\title{
Comment contenir davantage la hausse des primes
}

\author{
Jürg Schlup \\ Dr méd., président de la FMH
}

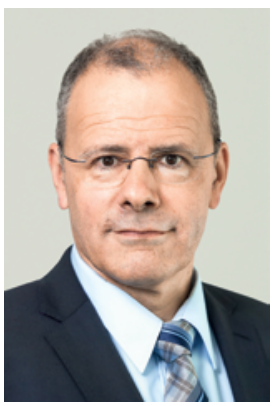

Communiqué de santésuisse du 12.7.2018. Hausse plus modérée des dépenses de santé en 2017. URL: http://www. santesuisse.ch/fr/detail/ content/hausse-plusmoderee-des-depensesde-sante-en-2017/

2 Communiqué de presse de la Confédération du 26.9.2016. En 2017, la prime standard augmentera de $4,5 \%$ en moyenne. URL: https://www. admin.ch/gov/fr/ accueil/documentation/ communiques.msgid-63902.html

3 gfs.bern. L'essentiel en bref sur le Moniteur de la santé 2018. Pas d'expériences souhaitées, mais plus

d'exigences vis-à-vis de la prise en charge. Interpharma, Bâle, 2018

4 Pour la période de 1997 à 2012 dans «L'essentiel en bref sur le Moniteur de la santé 2012»; pour la période de 2010 à 2016 dans "L'essentiel en bref sur le Moniteur de la santé 2016».

5 Golder, et al. Environnement de travail en mutation et attitude envers de nouveaux modèles de financement. Effets visibles de l'orientation vers la performance dans le domaine de la santé. Rapport final de gfs.bern étude concomitante sur mandat de la FMH. Janvier 2018

6 OBSAN. Analyse de l'enquête IHP (Inter-

national Health Policy) 2012 et 2015
Cette année, la discussion sur les primes entraîne dans son sillage un nombre inhabituel de bonnes nouvelles: avec une hausse en 2017 de 1,7\% seulement, l'augmentation des coûts de l'assurance obligatoire des soins (AOS) a été nettement inférieure à la moyenne pluriannuelle de 3,2\% [1], ce qui signifie aussi que la hausse des coûts a été très inférieure à l'augmentation des primes décidée pour 2017 (4,5\%) [2].

Une autre bonne nouvelle est que le nombre de personnes satisfaites de notre système de santé n'a encore jamais été aussi élevé (87\% de la population) [3]. En même temps, le nombre de personnes qui considèrent que les primes des caisses-maladie sont un problème récurrent $(5 \%)$ ou occasionnel $(11 \%)$ n'a jamais été aussi faible qu'aujourd'hui depuis l'introduction de la LAMal [3, 4]. En 2010, elles étaient plus du triple.

Pourtant, nous ne devons pas relâcher nos efforts pour améliorer notre système de santé et maintenir sa haute qualité au meilleur coût possible. Pour cela, la FMH préconise de suivre trois axes fondamentaux. Premièrement, un virage décisif: mettre en place un financement uniforme des prestations ambulatoires et stationnaires. C'est le seul moyen d'accroître le nombre de traitements ambulatoires, donc moins chers et moins lourds pour les payeurs de prime. Cela permettrait aussi de renforcer les soins intégrés et d'en exploiter le potentiel d'économie. Deuxièmement, le transfert renforcé de l'hospitalier vers l'ambulatoire exigera des adaptations rapides de nos structures hospitalières, telles qu'une planification supracantonale et des centres de chirurgie ambulatoire. Mais au préalable, il est indispensable de résoudre la question des conflits d'intérêts des cantons, dont les multiples rôles, comme propriétaires et planificateurs hospitaliers, mandataires et créanciers, instances de surveillance et d'approbation des tarifs, renforcent des déséquilibres.

Troisièmement, le fardeau administratif excessif offre un potentiel d'économie important. Un médecin hospitalier en soins somatiques aigus passe près d'un tiers de son temps à de l'administration [5]. Depuis 2011 en Suisse, la seule augmentation du temps consacré aux dossiers des patients impose chaque année cent nouveaux postes de médecins à plein temps. Les médecins installés ne sont pas épargnés non plus [6]. A la p. 1097, l'ASMAC montre comment les médecins travaillent de manière constructive à des améliorations. La deuxième phase de leur campagne intitulée «Plus de médecine et moins de bureaucratie!» vise à sensibiliser la Berne fédérale à ces questions importantes. Cette prise de conscience est essentielle pour une évolution efficace des coûts de notre système de santé, car,

\section{En 2017, les coûts de l'assurance obligatoire} des soins (AOS) ont augmenté de $1,7 \%$, soit nettement moins que les primes standard (4,5\%).

jusqu'à présent, la question des tâches administratives n’a que peu été prise en compte au niveau fédéral. La charge administrative n'est pratiquement pas mentionnée dans le rapport du groupe d'experts de 141 pages, bien que certaines des mesures évoquées soient liées à une augmentation de la bureaucratie. Un regard porté vers l'étranger montre pourtant que l'introduction d'un budget global impose encore plus de tâches administratives pour les médecins et exige des structures administratives importantes.

\section{Maîtriser les coûts passe par un financement} uniforme, une planification hospitalière supracantonale et une réduction des tâches administratives.

On peut douter qu'une administration accrue engendre plus de bénéfices pour les patients que ce qu'il leur en coûte. S'agissant de la prise en charge des patients, diverses études montrent en revanche que «les soins de santé d'aujourd'hui génèrent plus d'avantages pour les assurés que ce qu'ils paient pour leur assurance-maladie», comme l'explique Harry Telser, économiste de la santé, dans son article à la p. 1094. C'est aussi une bonne nouvelle pour la discussion sur les primes. 